\title{
Adverse drug effects following oseltamivir mass treatment and prophylaxis in a school outbreak of 2009 pandemic influenza A(H1N1) in June 2009, Sheffield, United Kingdom
}

M Strong (m.strong@sheffield.ac.uk) ${ }^{1}$, J Burrows ${ }^{1}$, E Stedman², P Redgrave ${ }^{3}$

1. School of Health and Related Research, University of Sheffield, Sheffield, United Kingdom

2. Medical School, University of Sheffield, Sheffield, United Kingdom

3. NHS Sheffield, Sheffield, United Kingdom

During the containment phase of the 2009 influenza $\mathrm{A}\left(\mathrm{H}_{1} \mathrm{~N}_{1}\right)$ pandemic, mass treatment and prophylaxis with oseltamivir was used to control an outbreak of pandemic influenza in a primary school in Sheffield, United Kingdom, where ten cases of pandemic influenza had been laboratory confirmed over a three day period in June 2009. A subsequent cross-sectional survey showed that 51 of 297 (17\%) pupils and 10 of $58(17 \%)$ reported an influenza-like illness. The most common symptoms were headache, cough, fever, tiredness, sore throat and nausea. Fifty-three staff and 273 pupils took oseltamivir for treatment or prophylaxis. Of this group, $41 \%(113 / 273)$ of pupils and $47 \%(25 / 53)$ of staff reported adverse effects. Overall, $14 \%(37 / 273)$ of pupils and $20 \%(11 / 53)$ of staff did not complete the course of oseltamivir, primarily due to adverse effects. Nausea, vomiting and rash were statistically significantly associated with failing to complete the course of oseltamivir. Given the potential for side effects from oseltamivir, particularly among those without influenza who receive the drug for prophylaxis, our findings have two important implications. Firstly, the benefits of mass treatment in an outbreak setting must clearly be greater than the benefits of targeted treatment. Secondly, any large scale regional or state level system for distribution of antiviral drugs for treatment should ideally include a robust quantification of an individual's probability of infection with influenza virus in order to avoid unnecessary treatment.

\section{Introduction}

An outbreak of 2009 pandemic influenza $A\left(\mathrm{H}_{1} \mathrm{~N}_{1}\right)$ occurred in a Sheffield junior school in June 2009, during the United Kingdom pandemic influenza containment phase. Over a three day period a seasonally unusual number of pupils and staff became unwell with influenza-like illness. Ten cases were laboratory confirmed as pandemic influenza and a decision was taken to close the school for one week and offer oseltamivir (unless contraindicated) to pupils and staff regardless of their being symptomatic or not.

As with any drug, oseltamivir is associated with adverse effects. In clinical trials including adults and adolescents with influenza, nausea and vomiting were statistically significantly more common in those who took the treatment dose of oseltamivir compared with those who took placebo ( $11 \%$ versus $7 \%$ for nausea alone, and $8 \%$ versus $3 \%$ for vomiting) [1]. Anecdotal evidence at the time of the outbreak described, suggested that the prevalence of adverse gastrointestinal effects could be somewhat higher than this, and subsequent studies in other outbreaks with pandemic influenza in England have confirmed this [2,3].

The prevalence and severity of adverse drug reactions, whether perceived or experienced, are important factors in patients' adherence to medication, and adherence is improved if the benefit of a drug is perceived to outweigh potential harm [4]. The balance between benefit and potential harm is particularly important where a drug is taken prophylactically for a disease that is considered to be mild. In such situations harm may easily outweigh benefit and lead to poor compliance. Poor compliance with prophylaxis in the context of a communicable disease outbreak is of public health concern if the drug is being used not only to protect the individual from disease, but also to reduce person-toperson transmission.

Our study aimed to determine the prevalence of adverse effects from treatment and from prophylaxis with oseltamivir, and whether these were associated with failure to complete the course of oseltamivir. 


\section{Methods}

The school was closed at the end of the school day on Friday, 12 June and reopened on the morning of Monday, 22 June. Oseltamivir was offered to all staff and pupils at the school by local NHS Sheffield and Health Protection Agency staff. The medication was distributed at the school on the evening of 12 June, and the morning of 13 June. Those reporting fever plus two other influenza associated symptoms were considered clinical cases of influenza, and were prescribed an age appropriate treatment dose $(60 \mathrm{mg}$ for school pupils and $75 \mathrm{mg}$ for adults) twice daily for five days. Laboratory confirmation was not routinely undertaken on the clinical cases reported during the mass treatment phase of the outbreak. Those who did not fit this case definition were prescribed the same dose as for treatment, but once daily for 10 days.

\section{Questionnaire}

We conducted a cross-sectional survey and approximately two weeks after the end of the outbreak, a questionnaire was distributed via the school to all pupils and staff to be filled in anonymously. The questionnaire asked for the following information: whether or not the respondents had been 'poorly with flu symptoms', which symptoms they had experienced, any past medical history, whether they had taken oseltamivir and how long they had taken it for, any adverse effects they had experienced, and if they had stopped taking oseltamivir, why had they done so. Finally, respondents were asked if there was anything else they would like to tell us (Figure 1).

Figure 1. Questionnaire on influenza-like illness and oseltamivir use, school outbreak of 2009 pandemic influenza $A\left(\mathrm{H}_{1} \mathrm{~N}_{1}\right)$, Sheffield, June 2009 (se attached questionnaire).

\section{Statistical analysis}

Data were analysed in $\mathrm{R}$ 2.9.1 [5]. For proportions we calculated $95 \%$ confidence intervals $(\mathrm{Cl})$ using the exact binomial method. Association between failure to complete the course of oseltamivir and the presence or absence of adverse effects was explored using multivariate logistic regression. We retained in the model

\section{FIGURE 2}

Epidemic curve of cases of influenza-like illness by onset date, school outbreak of 2009 pandemic influenza A(H1N1), Sheffield, June 2009

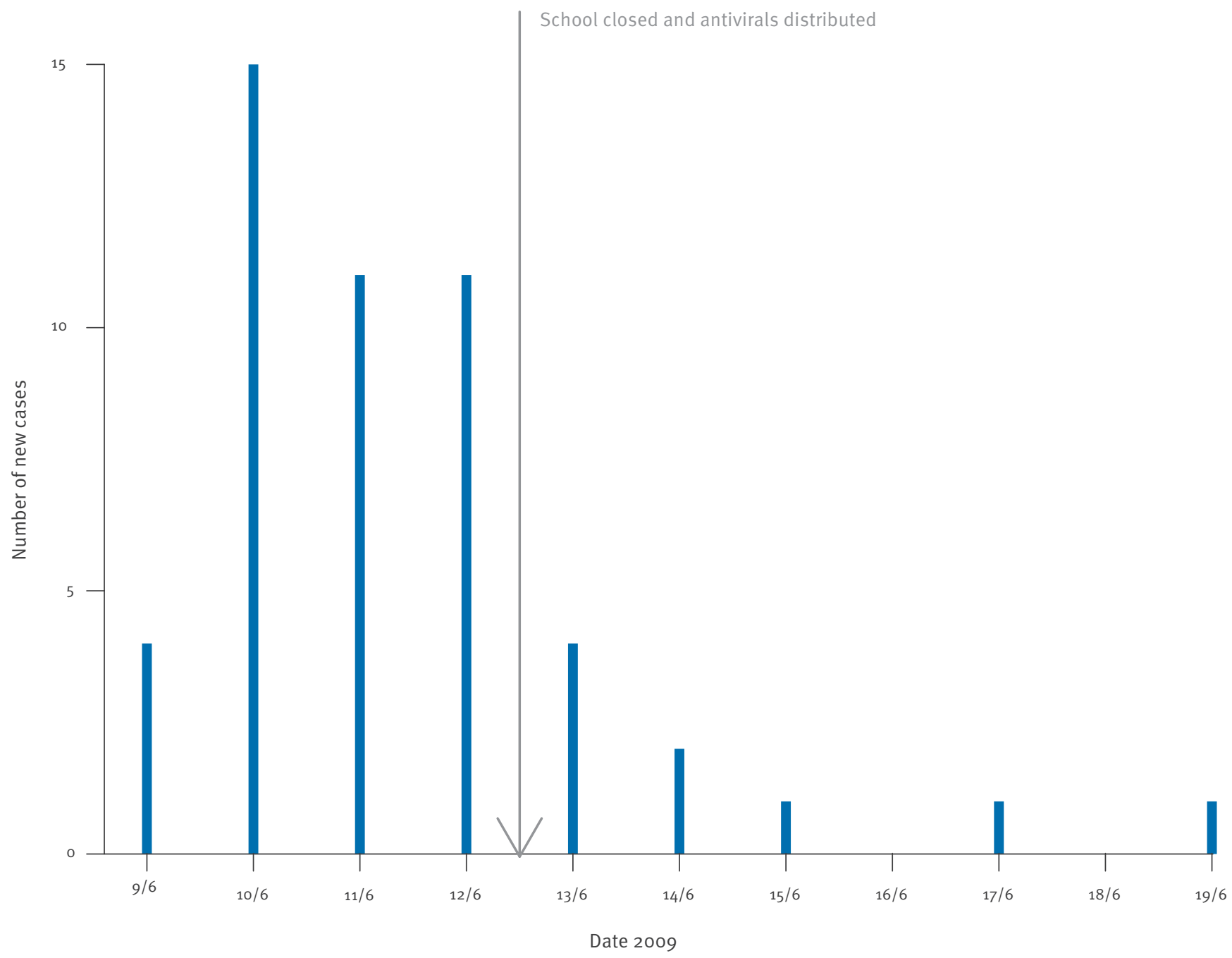


TABLE 1

Symptoms $s^{\mathrm{a}, \mathrm{b}}$ in people reporting influenza-like illness, school outbreak of 2009 pandemic influenza A(H1N1), Sheffield, June 2009 ( $n=61)$

\begin{tabular}{|l|c|c|c|}
\hline Symptom & $\mathbf{n}$ & $\mathbf{\%}$ & $\mathbf{9 5} \% \mathbf{C I}$ \\
\hline Headache & 51 & 84 & $72-92$ \\
\hline Cough & 49 & 80 & $68-89$ \\
\hline Tiredness & 42 & 69 & $56-80$ \\
\hline Fever & 42 & 69 & $56-80$ \\
\hline Sore throat & 32 & 52 & $39-65$ \\
\hline Nausea & 31 & 51 & $38-64$ \\
\hline Shivery & 29 & 47 & $35-60$ \\
\hline Runny nose & 28 & 46 & $33-59$ \\
\hline Aching & 27 & 44 & $32-58$ \\
\hline Abdominal pain & 26 & 43 & $30-56$ \\
\hline Dizziness & 22 & 36 & $24-49$ \\
\hline Leg ache & 18 & 29 & $18-43$ \\
\hline Vomiting & 12 & 20 & $11-32$ \\
\hline Breathing problems & 8 & 13 & $6-24$ \\
\hline Diarrhoea & 8 & 13 & $6-24$ \\
\hline
\end{tabular}

$\mathrm{Cl}$ : confidence interval.

a Symptoms reported in more than $10 \%$ of people are listed.

${ }^{\mathrm{b}}$ More than one symptom may be listed per person.

\section{TABLE 2}

Adverse effects reported by $>1 \%$ of people taking oseltamivir, school outbreak of 2009 pandemic influenza A(H1N1), Sheffield, June 2009 (n=326)

\begin{tabular}{|l|c|c|c|}
\hline Adverse effect & $\mathbf{n}$ & $\mathbf{\%}$ & $\mathbf{9 5} \% \mathbf{C l}$ \\
\hline Nausea & 86 & 26 & $22-32$ \\
\hline Abdominal pain & 64 & 20 & $15-24$ \\
\hline Headache & 38 & 12 & $8-16$ \\
\hline Dizziness & 28 & 9 & $6-12$ \\
\hline Diarrhoea & 25 & 8 & $5-11$ \\
\hline Vomiting & 22 & 7 & $4-10$ \\
\hline Insomnia & 19 & 6 & $3-9$ \\
\hline Sore eyes & 6 & 2 & $1-4$ \\
\hline
\end{tabular}

\section{TABLE 3}

Adverse effects reported by $<1 \%$ of people taking oseltamivir, school outbreak of 2009 pandemic influenza A(H1N1), Sheffield, June 2009 ( $n=326)$

\begin{tabular}{|l|l|}
\hline Adverse effect & $\mathbf{n}$ \\
\hline Rash & 3 \\
\hline Nose bleed & 2 \\
\hline Back ache & 1 \\
\hline Itch & 1 \\
\hline Blurred vision & 1 \\
\hline Irritability & 1 \\
\hline Mood swings & 1 \\
\hline “Spaced out” & 1 \\
\hline Anxiety & 1 \\
\hline Frustration & 1 \\
\hline Tearful & 1 \\
\hline
\end{tabular}

those adverse effect covariates that were significantly associated, at p<0.05 level, with failure to complete the course. For the included adverse effect covariates we present odds ratios (OR) as the measure of association, along with $95 \% \mathrm{Cl}$.

We tested for a difference in the proportions of respondents with and without influenza-like illness reporting adverse effects using a chi-square test for homogeneity with a null hypothesis of no difference and a significance level of pro.05. We tested for a difference in the proportions of respondents with and without influenza-like-illness who failed to complete the course of oseltamivir in the same way.

\section{Results}

The epidemic curve shows the number of new cases of influenza-like illness by day over the period of the outbreak from June 9 to June 19, 2009; fewer cases occurred with a reported onset date after the school closure compared with the time before (Figure 2).

The response rate to the questionnaire was $84 \%$ (58/69) among staff and $62 \%(297 / 476)$ among pupils. Pupils who responded were between seven and twelve years old with a mean age of 9.5 years. Staff were asked to indicate which 10-year age band they fell in and 64\% (37/58) staff were aged between 40 and 59. Of the 341 respondents who indicated their sex, $45 \%(129 / 288)$ of pupils and $91 \%(48 / 53)$ of staff were female.

\section{Influenza-like illness}

Influenza-like illness was reported by 51 pupils (17\%; $95 \% \mathrm{Cl}: 13-22 \%)$ and 10 staff (17\%; $95 \% \mathrm{Cl}: 9-29 \%)$. Pupils who indicated that they were 'generally fit and well' in terms of their overall health were less likely to report that they had had influenza $(\mathrm{OR}=0.45 ; 95 \% \mathrm{Cl}$ : 0.22-0.98; $p=0.038)$. In staff, no such association was seen.

\section{TABLE 4}

Reasons for not taking oseltamivir, school outbreak of 2009 pandemic influenza A(H1N1), Sheffield, June 2009 $(n=29)$

\begin{tabular}{|l|c|}
\hline Reason & $\mathbf{n}$ \\
\hline I didn't think it was needed & 13 \\
\hline Contraindicated & 3 \\
\hline I thought it would make me sick & 2 \\
\hline I was worried about taking it & 1 \\
\hline Worried about side effects & 1 \\
\hline I don't like taking medicines & 1 \\
\hline GP advised me not to take it & 1 \\
\hline Already take other medication & 1 \\
\hline Would take it only if sick with flu & 1 \\
\hline In a different year to those affected & 1 \\
\hline Doesn't stop flu, only reduces length of symptoms & 1 \\
\hline
\end{tabular}

GP: general practioner. 
The most common symptoms reported by the 61 persons with influenza-like illness, were headache $(84 \%$; 95\% Cl: 72-92), cough (80\%; 95\% Cl: 68-89), fever (69\%; 95\% Cl: 56-80), tiredness (69\%; $95 \% \mathrm{Cl}: 56-80$ ), sore throat (52\%; 95\% Cl: $39-65)$ and nausea (51\%; 95\% Cl: 38-64) (Table 1).

\section{Uptake, adverse effects and}

\section{adherence to oseltamivir}

Fifty-three staff (91\%; 95\% Cl: 81-97) and 273 pupils (92\%; $95 \% \mathrm{Cl}: 88-95)$ took up the offer of taking oseltamivir.

Adverse effects were reported by 113 pupils (41\%; 95\% $\mathrm{Cl}: 35-47)$ and 25 staff (47\%; 95\% Cl: 33-61). The most commonly reported adverse effects, mentioned by over $10 \%$ of the sample were nausea ( $26 \%$; $95 \% \mathrm{Cl}: 22-32$ ), abdominal pain (20\%; $95 \% \mathrm{Cl}: 15-24)$, and headache (12\%; 95\% Cl: $8-16)$ (Table 2 ). While the majority of adverse effects were physical in nature, two respondents, one staff and one pupil, reported a number of symptoms related to disturbed mood and 19 reported insomnia. There were no life-threatening adverse effects reported (Table 3 ).

Among those with influenza-like illness, 46\% (95\% $\mathrm{Cl}: 31-61)$ of pupils and $56 \%(95 \% \mathrm{Cl}: 21-86)$ of staff reported adverse effects. In those who were well, $40 \%$ (95\% Cl: $34-47)$ of pupils and $45 \%$ (95\% Cl: $30-61)$ of staff reported adverse effects. There was no significant difference in the proportion reporting adverse effects between those with influenza-like illness and those without (chi-square $=0.26, d f=1, p=0.6$ ), or between pupils and staff (chi-square $=0.33, \mathrm{df}=1, \mathrm{p}=0.57$ ).
Thirty-seven pupils (14\%; 95\% Cl: 10-18) and 11 staff (20\%; $95 \% \mathrm{Cl}: 11-34)$ did not complete the full course of oseltamivir. The most common reasons given were 'it made me feel ill' (50\%; 95\% Cl: 35-65), 'I didn't think it would help' (17\%; $95 \% \mathrm{Cl}: 7-30)$ and 'I forgot to take it' (15\%; $95 \% \mathrm{Cl}: 6-28)$. In a logistic regression model, stopping oseltamivir was significantly associated with the presence of nausea $(\mathrm{OR}=2.4 ; 95 \% \mathrm{Cl}: 1.2-4.9$; $\mathrm{p}=0.013)$, vomiting $(\mathrm{OR}=3.5 ; 95 \% \mathrm{Cl}: 1.3-9.3 ; \mathrm{p}=0.014)$ and rash $(\mathrm{OR}=13.0 ; 95 \% \mathrm{Cl}: 1.1-299.3 ; \mathrm{p}=0.046)$. There was no significant difference in the proportion between those with influenza-like illness and those without (chi-square $=0.062 ; \mathrm{df}=1 ; \mathrm{p}=0.8$ ), or between pupils and staff (chi-square $=0.58 ; d f=1 ; p=0.45$ ).

Among those with influenza symptoms who did not complete a full course of oseltamivir the median number of days taking the drug was three (interquartile range (IQR) 3-4.5). Among those without influenza-like illness who did not complete a full course of oseltamivir the median number of days taking the drug was seven (IQR 5-8).

Reasons for choosing not to take oseltamivir Twenty-four pupils ( $8 \% ; 95 \% \mathrm{Cl}: 5-12)$ and five staff (9\%; 95\% Cl: 3-19) chose not to take any oseltamivir. The most common reason given was 'I didn't think it was needed' (45\%; 95\% Cl: 26-64\%) (Table 4).

\section{Discussion and conclusion}

In a primary school outbreak of 2009 pandemic influenza $A\left(\mathrm{H}_{1} \mathrm{~N}_{1}\right), 41 \%$ of pupils and $47 \%$ of staff who took oseltamivir for either treatment or prophylaxis reported adverse effects. Overall, 48 of 355 (15\%) pupils and staff stopped taking oseltamivir, primarily due to adverse effects. Nausea, vomiting and rash

\section{TABLE 5}

Prevalence of adverse effects in people taking oseltamivir prophylactically

\begin{tabular}{|c|c|c|c|c|c|c|}
\hline \multirow{3}{*}{ People affected } & \multirow{3}{*}{ Adverse effect } & \multicolumn{5}{|c|}{ Prevalence (\%) } \\
\hline & & \multicolumn{2}{|c|}{ Product characteristics ${ }^{a}$} & \multirow{2}{*}{ 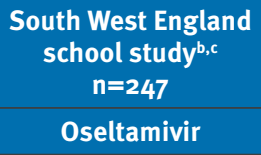 } & \multirow{2}{*}{$\begin{array}{c}\begin{array}{c}\text { London schools } \\
\text { study } \\
\text { n=e }\end{array} \\
\text { Ose } \\
\text { Oseltamivir }\end{array}$} & \multirow{2}{*}{$\begin{array}{c}\begin{array}{c}\text { Sheffield studyi,g } \\
n=326\end{array} \\
\text { Oseltamivir }\end{array}$} \\
\hline & & Oseltamivir & Placebo & & & \\
\hline \multirow[t]{4}{*}{$\begin{array}{l}\text { Children aged up to } \\
12 \text { years }\end{array}$} & Nausea & 14 & - & 33 & 29 & 23 \\
\hline & Abdominal pain & 1 & - & 21 & 16 & 20 \\
\hline & Diarrhoea & 1 & - & 7 & 0 & 6 \\
\hline & Vomiting & 10 & - & 11 & 13 & 7 \\
\hline \multirow[t]{4}{*}{ Adults and adolescents } & Nausea & 10 & 4 & - & 30 & 31 \\
\hline & Abdominal pain & 2 & 2 & - & 22 & 8 \\
\hline & Vomiting & 2 & 1 & - & 4 & 6 \\
\hline & Insomnia & 1 & 1 & - & 15 & 6 \\
\hline
\end{tabular}

a Source: [1].

b Source [2].

c Pupils were aged 11-12 years (school year 7).

d Source [3].

e Prevalence among pupils from both secondary schools included under Adults and adolescents.

f Source: this study (school outbreak of 2009 pandemic influenza $A\left(\mathrm{H}_{1} \mathrm{~N}_{1}\right)$, Sheffield, June 2009).

${ }^{g}$ Prevalence among staff included under Adults and adolescents. 
were statistically significantly associated with failing to complete the course of oseltamivir.

We were able to distribute our questionnaire to an entire school population who had been offered oseltamivir irrespective of symptoms, and our response rate was high among staff (84\%), and moderately high among pupils $(62 \%)$. We received a considerable number of positive comments regarding the handling of the outbreak in response to the open question 'anything else you would like to tell us'. This suggests that there was general support for our intervention in the school. However, as with any study that does not have a $100 \%$ response rate, we cannot fully exclude that those who did not respond may have been systematically different from those who did. In particular, we might expect those who had experienced drug-associated adverse effects to be more likely to participate than those who had not, introducing a bias in our findings. Moreover, one difficulty with our questionnaire (and with this type of observational study in general) is in determining whether symptoms in those who reported influenza-like illness were a result of the oseltamivir used to treat the illness, or the disease itself. Furthermore, we did not ask about the severity of any influenza-like symptoms or adverse effects experienced, and to some extent this is a limitation of our study as we are unable to test for a relationship between oseltamivir dose (expressed, ideally, as dose per unit of child's weight) and the severity of the adverse effect. We were, however, able to determine through our contact with local paediatric services that there had been no reports of any life threatening or serious adverse effects associated with oseltamivir use in our cohort.

Due to the difficulties of obtaining accurate retrospective information from junior school aged children we do not know the degree to which our results may have been biased. We tried to reduce any such bias by asking parents to assist their children in filling the questionnaire, but we are aware that obtaining accurate information can be a problem in retrospective questionnaire studies, irrespective of the age of the respondents. In order to maximise the accuracy of the recall of symptoms and adverse effects we conducted our study as soon as possible following the reopening of the school.

\section{Our study in the context of previous studies}

Two studies similar to ours have been published recently in the United Kingdom, one following an outbreak of 2009 pandemic influenza in a school in South West England [2], and the other following outbreaks in three London schools [3]. The South West study reported adverse effects associated with oseltamivir and compliance with prophylaxis in 11-12 year old pupils in a single school year, whereas the London study involved pupils from one primary school (4- to 11-year-olds), and two secondary schools (11- to 14-year-olds).

The findings of our study are broadly consistent with those found in the two studies. Adverse effects were reported by $51 \%$ of 247 pupils in the South West, and $53 \%$ of 85 pupils in London, compared with $41 \%$ in our study. The majority of children in the previous studies were of secondary school age, and therefore older than our cohort. It is possible that older children are more likely to either experience, or report experiencing adverse effects following the use of oseltamivir. Interestingly, a trend in our study showed that a greater proportion of adults reported adverse effects than children, although this difference was not statistically significant.

The prevalence of adverse effects associated with a drug is reported in its Summary of Product Characteristics (SPC). The SPC for oseltamivir draws on adverse event data from treatment and prophylaxis trials and from post marketing surveillance [1]. Table 5 shows the comparative prevalence of adverse reactions (for those reactions reported in all studies) between the SPC and the school based studies amongst those taking oseltamivir for prophylaxis.

The school based studies fairly consistently report a higher prevalence of adverse effects than the trials that informed the SPC. This may be due to the inevitable differences in methods for eliciting adverse event information between highly structured and regulated clinical trials and more informal questionnaire surveys. It is possible that the presence of 'tick box' options for reporting a number of specific adverse reactions may have encouraged over reporting in our survey and also could have favoured symptoms and adverse effects listed over those not listed. Moreover, the anxiety generated by the arrival of a pandemic may have led to a greater attention towards adverse effects.

Reported adherence to oseltamivir for prophylaxis varied between the three school studies, with $66 \%$ of pupils in the London schools completing the full ten day course compared with $80 \%$ in the South West. Of the 271 asymptomatic pupils in our study 230 (85\%) completed the full ten day course. It could be that older children are less likely to complete a course of medication, although we did not find this age effect within our data.

\section{Conclusion}

In our study, conducted in the context of a school outbreak of 2009 pandemic influenza, mass treatment and prophylaxis with oseltamivir was associated with adverse effects in a considerable proportion of pupils and staff. Despite this, adherence to the antiviral medication regime was generally good. Given the potential for side effects from oseltamivir, particularly among those without influenza who receive the drug for prophylaxis, our findings have two important implications. Firstly, the benefits of mass treatment in an outbreak setting must clearly be greater than the benefits of targeted treatment. Secondly, any large scale regional or state level system for distribution of antiviral drugs for treatment should ideally include a robust quantification of an individual's probability of infection with influenza virus in order to avoid unnecessary treatment. 


\section{Acknowledgements}

We would like to thank all the staff and pupils at the school concerned for their contribution to the survey.

\section{References}

1. Roche Products Limited. Summary of product characteristics for Tamiflu. The electronic Medicines Compendium (eMC).

Hertfordshire: 2009. Available from: http://emc.medicines.org. uk/document.aspx?documentld=10446

2. Wallensten A, Oliver I, Lewis D, Harrison S. Compliance and side effects of prophylactic oseltamivir treatment in a school in South West England. Euro Surveill. 2009;14(30). pii=19285. Available from: http://www.eurosurveillance.org/ViewArticle. aspx?Articleld $=19285$

3. Kitching A, Roche A, Balasegaram S, Heathcock R, Maguire $H$. Oseltamivir adherence and side effects among children in three London schools affected by influenza $A\left(\mathrm{H}_{1} \mathrm{~N}_{1}\right) \mathrm{v}$, May 2009 - an internet-based cross-sectional survey . Euro Surveill. 2009;14(30). pii=19287. Available from: http://www. eurosurveillance.org/ViewArticle.aspx?Articleld $=19287$

4. World Health Organisation. Adherence to Long term Therapies: Evidence for Action. Geneva: World Health Organization; 2003:30. Available from: http://www.who.int/chp/knowledge/ publications/adherence_report/en/index.html

5. R Development Core Team. R: A language and environment for statistical computing. R Foundation for Statistical Computing, Vienna: 2009. Available from: http://www.R-project.org. 
PUPIL QUESTIONNAIRE: Please return to school by

Thank you for helping us find out about any flu symptoms you may have had and any side effects from the Tamiflu medicine that you were given.

Age: Class: Sex: Male / Female

Q1 Were you poorly with flu symptoms?

YES

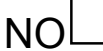

If YES please go to Q2. If NO please go to Q4

Q2 When did you start feeling poorly?

Q3 If you were poorly, did you have any of the following symptoms?

(Please tick all that apply)

A fever

Feeling shivery

Tummy ache

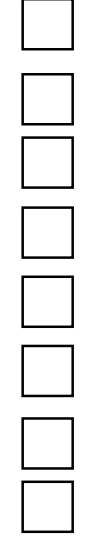

Cough

Sore knees

Feeling sick

Breathing problems

Being sick

Runny nose

Diarrhoea

Headache

Leg ache

Aching all over

Feeling very tired

Feeling dizzy

Sore throat

Any other symptoms

What was your highest temperature (if measured)?

Which smiley face best shows how you felt when you were most poorly?

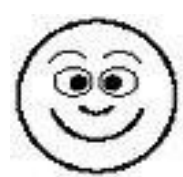

0

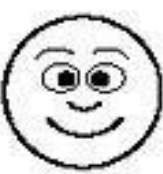

1

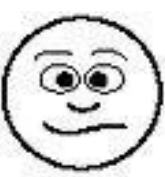

2

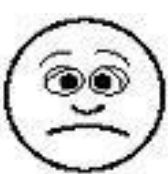

3

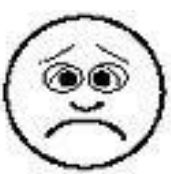

4

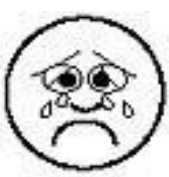

5

Q4 Did you have a positive swab result for swine flu? YES

NO

Q5 Are you generally fit and well?

Please tell us if you have any have any ongoing health problems such as asthma, diabetes etc 
PUPIL QUESTIONNAIRE: Please return to school by

Q6 Were you given any Tamiflu tablets?

YES

NO

Q7 Did you take the Tamiflu? YES

NO $\square$ YES BUT STOPPED

How many days did you take it for

How many times a day did you take it?

If NO, please tick which reasons apply:

(These may be your own or your parents' reasons)

I was worried about taking it

$\square$ I didn't think it would work

I don't like taking medicines

$\square$ I thought it would make me sick

I didn't think it was needed

$\square$ Other people weren't taking it

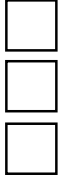

Other reasons

If STOPPED, please tick which reasons apply:

It made me feel ill

I forgot to take it

$\square$ I didn't think it was helping
$\square \quad$ I heard bad things about it

Other reasons

Q8 If you took Tamiflu did it make you feel poorly? YES $\square$ NO

If YES did you have any of the following? (Please tick all that apply)

Tummy ache

Feeling sick

Being sick

Diarrhoea

Headache

Nose bleed

Sore eyes
Trouble sleeping

Back ache

Rash

Feeling dizzy

Please describe any other symptoms:

Q9 Is there anything else you would like to tell us?

If you want to talk to someone about this questionnaire, please phone the Health Protection Unit on $x x x x x x x x x x x$. For other enquiries about swine flu please phone $x x x x$ xxxxxxxx. If you are unwell please phone NHS Direct on $x x x x$ xx xx or contact your GP. 